EPJ Web of Conferences 79, 02002 (2014)

DOI: $10.1051 /$ epjconf/20147902002

(C) Owned by the authors, published by EDP Sciences, 2014

\title{
HELNED: Helium-3-free low-cost neutron detectors
}

\author{
Massimo Barbagallo ${ }^{1}$, Luigi Cosentino ${ }^{2}$, Carmelo Marchetta ${ }^{2}$, Alfio Pappalardo ${ }^{2}$, Carlotta Scirè2, \\ Sergio Scirè ${ }^{2}$, Gianfranco Vecchio ${ }^{2}$ and Paolo Finocchiaro ${ }^{2, a}$ \\ ${ }^{1}$ INFN Sezione di Bari, via Orabona 4, 70126 Bari, Italy \\ ${ }^{2}$ INFN Laboratori Nazionali del Sud, via S.Sofia 62, 95125 Catania, Italy
}

\begin{abstract}
We developed a technique for thermal neutron detection not making use of ${ }^{3} \mathrm{He}$, also suitable for online real time monitoring of CASTOR spent fuel containers. As a neutron converter we used ${ }^{6} \mathrm{LiF}$, being the neutron capture cross section of ${ }^{6} \mathrm{Li}$ very well known and with only an alpha and a triton in the exit channel. We can deposit thin layers of converter onto several different substrates, to be placed on top of solid state detectors or scintillators capable of efficiently detecting the decay products.
\end{abstract}

\section{Introduction}

For the detection of thermal or low energy neutrons, as a viable alternative to ${ }^{3} \mathrm{He}$ tubes $[1,2]$, semiconductor-based detectors can be used in combination with a neutron reactive film, usually called neutron converter, which converts neutrons into charged particles.

The main characteristics of a converter, suitable for use in combination with a solid state detector, are large neutron absorption cross section, small gamma ray emission, and presence of decay channels into energetic charged particles. The two nuclear species commonly considered suitable neutron converters are ${ }^{6} \mathrm{Li}$ (natural abundance $\approx 7 \%$, thermal neutron cross section $\approx 940 \mathrm{~b}$ ) and ${ }^{10} \mathrm{~B}$ (natural abundance $\approx 20 \%$, thermal neutron cross section $\approx 3840 \mathrm{~b}$ ). The corresponding neutron capture reactions involved are:

$$
{ }^{6} \mathrm{Li}+n \rightarrow{ }^{3} \mathrm{H}(2.73 \mathrm{MeV})+\alpha(2.05 \mathrm{MeV})
$$

and

$$
\begin{aligned}
& { }^{10} \mathrm{~B}+n \rightarrow{ }^{7} \mathrm{Li}(1.01 \mathrm{MeV})+\alpha(1.78 \mathrm{MeV}) \text { ground state, } \mathrm{BR} \approx 6 \% \\
& { }^{10} \mathrm{~B}+n \rightarrow{ }^{7} \mathrm{Li}(0.84 \mathrm{MeV})+\alpha(1.47 \mathrm{MeV})+\gamma(0.48 \mathrm{MeV}) \text { excited state, } \mathrm{BR} \approx 94 \% .
\end{aligned}
$$

We decided to choose ${ }^{6} \mathrm{Li}$ because after capturing a neutron it has a unique decay channel, with no gamma rays, with a higher available kinetic energy and with lighter particles produced, therefore easier to detect than in the case of ${ }^{10} \mathrm{~B}$. We use it in form of $\mathrm{LiF}$, enriched at $95 \%$ in ${ }^{6} \mathrm{Li}$, that is a very stable and nonexpensive salt which we can deposit uniformly as very thin layers onto rather large substrates up to several hundreds of $\mathrm{cm}^{2}$.

\footnotetext{
${ }^{a}$ Corresponding author: finocchiaro@lns.infn.it
}

This is an Open Access article distributed under the terms of the Creative Commons Attribution License 4.0, which permits unrestricted use, distribution, and reproduction in any medium, provided the original work is properly cited. 


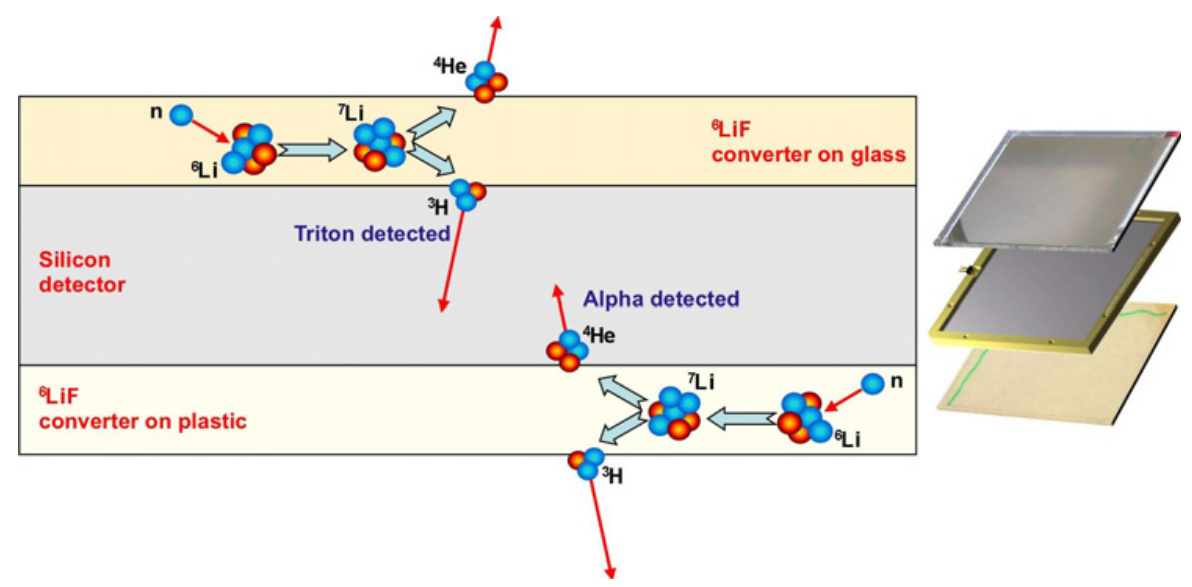

Figure 1. Sketch of a thermal neutron detector consisting of a double-sided silicon detector coupled with two ${ }^{6} \mathrm{LiF}$ neutron converters. For test purposes two different substrates were used: plastic and glass. The converter layer thickness was respectively $1.8 \mu \mathrm{m}$ and $1.5 \mu \mathrm{m}$. As the neutron energy is very low, the reaction products are emitted in opposite directions in the laboratory frame, and only one is detected.

Based on these assumptions in this paper we will show that cheap solid state neutron detectors with good n/gamma discrimination can be built, even though having a low intrinsic efficiency. We will also provide hints on how to improve such efficiency, also indicating a few strategies to set up similar detectors using scintillators. The choice of using independent detectors and converters allows a better modularity, partial replacements and reconfigurability.

Even though featuring low efficiency, these detectors can be fruitfully exploited for permanent realtime online monitoring of nuclear spent fuel casks. Thus they can represent a viable tool to ensure the continuity of knowledge after sealing the casks, that is the main ingredient both for safety and security.

\section{A silicon pad for neutron detection}

The charged particles lose some of their energy passing through the converter; the energy loss depends on the converter material itself, the particle type and its energy, and therefore the choice of film thickness that can be practically used is limited. Moreover, the reaction products entering the detector must have sufficient energy in order to produce signals large enough for particle discrimination from the gamma background.

The dependence of the spectrum shape on the converter thickness has been observed in several papers [3-7] where it is evident that a thinner film ensures a better separation between the reaction products and the background, although the detection efficiency becomes smaller.

In Fig. 1 we sketched the conversion mechanism of a neutron inside the ${ }^{6} \mathrm{LiF}$ layer. As the two reaction products are emitted in opposite directions, only one of them may enter the sensitive layer and be detected. The case shown makes use of a double-sided silicon pad detector, $3 \mathrm{~cm} \times 3 \mathrm{~cm}, 300 \mu \mathrm{m}$ thick, with a neutron converter on each face. In our tests, in view of investigating different configurations, we employed two slightly different converter thicknesses and substrates, namely $1.8 \mu \mathrm{m}$ on glass and $1.5 \mu \mathrm{m}$ on plastic.

The ${ }^{6} \mathrm{LiF}$ depositions on the substrates were done in house, using an evaporator complex, and the choice of having the converter independent of the detector allows an efficient modularity and operation.

In Fig. 2 we show a picture of a detector prototype, which was sandwiched between the two converters and then placed inside a neutron moderator made from polyethylene blocks (Fig. 3), in order 


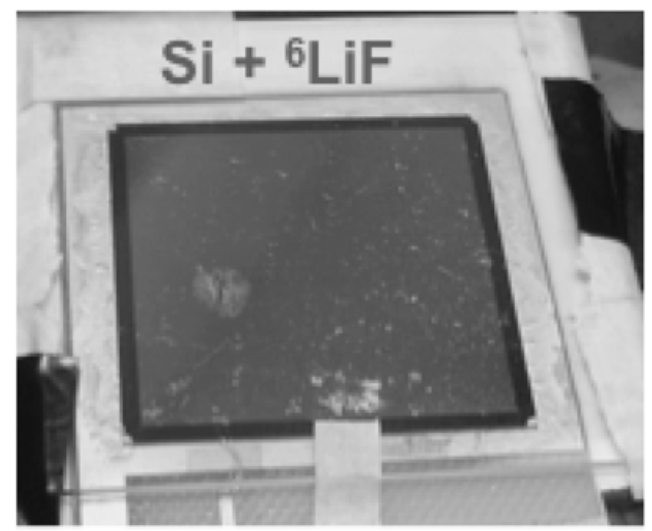

Figure 2. The silicon detector sandwiched between two ${ }^{6} \mathrm{LiF}$ neutron converters.

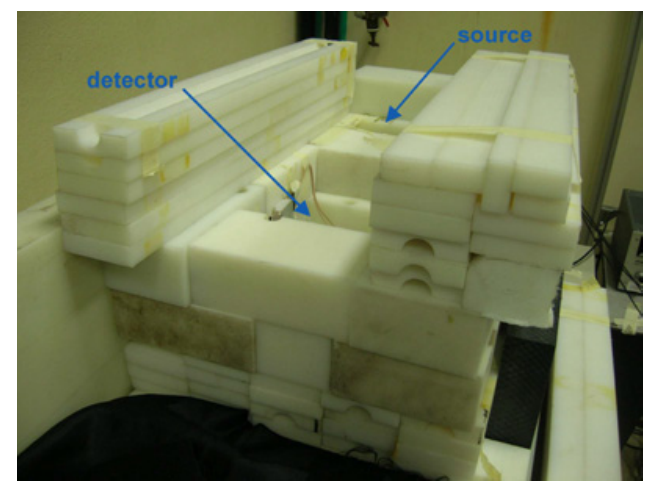

Figure 3. The neutron moderator made from polyethylene blocks.

to be tested with an AmBe neutron source. We remark that such a source, besides producing neutrons with a broad spectrum centered around $4-5 \mathrm{MeV}$, produces also as many high energy gamma rays $(\approx 4.4 \mathrm{MeV})$ as well as a huge amount of $\approx 59 \mathrm{KeV}$ low energy gamma rays. We performed such a test, under different configurations, in order to disentangle the detector behavior. In Fig. 4 we superimposed three typical spectra obtained with the detector under the following respective conditions: no neutron source (area 1), converters installed; with neutron source but no converter (area 2); with neutron source, converters installed (area 3). What immediately sticks out is that the spectrum without source, only due to background gamma (and cosmic) rays, lays entirely below about $0.3 \mathrm{MeV}$.

When exposing the detector without neutron converters to the AmBe source, the only contribution to the spectrum comes from gamma rays. In this case we can see that such a contribution lays below $\approx 0.5-0.6 \mathrm{MeV}$. Conversely, when installing the two converters and exposing the sandwich to the neutron source, we see a totally different spectrum shape, due to the detection of alpha and triton particles, coming from the neutron capture on ${ }^{6} \mathrm{Li}$ followed by the decay of ${ }^{7} \mathrm{Li}$ into alpha and triton. The alpha contribution in the spectrum is between $0.6 \mathrm{MeV}$ and $1.7 \mathrm{MeV}$, whereas the triton contribution is between $1.7 \mathrm{MeV}$ and $2.7 \mathrm{MeV}$, exactly as expected from calculations and simulations, and both represent a perfect signature of a neutron detection.

The macroscopic cross section of ${ }^{6} \mathrm{LiF}$ (enriched in ${ }^{6} \mathrm{Li}$ at $95 \%$ as it is our case) for thermal neutron capture is $57 \mathrm{~cm}^{-1}$, which for our converters $1.8 \mu \mathrm{m}$ and $1.5 \mu \mathrm{m}$ thick corresponds to intrinsic efficiency 


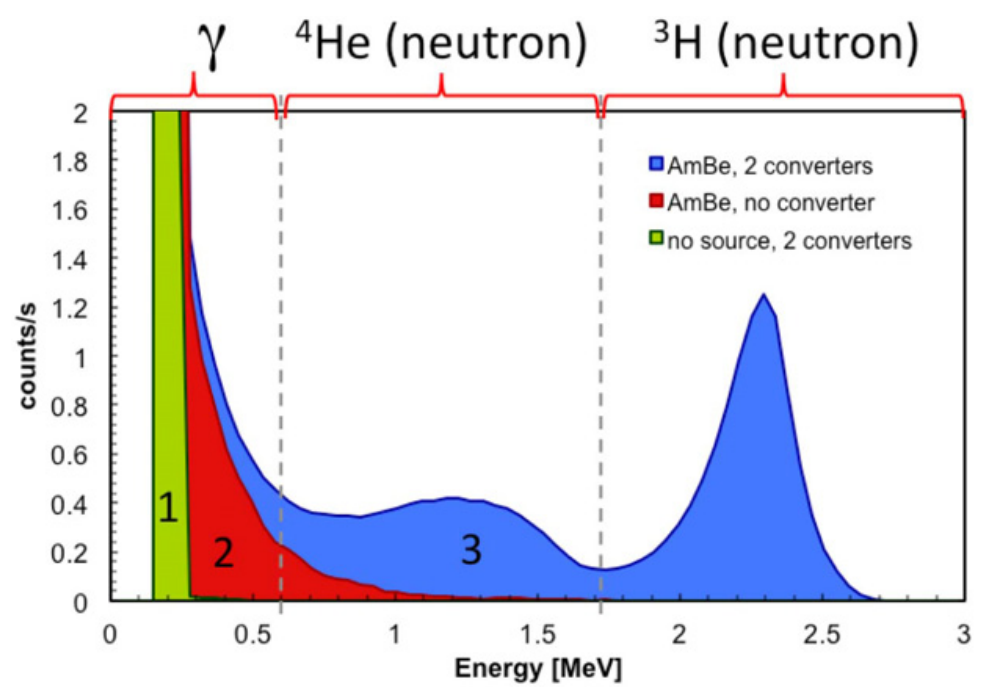

Figure 4. Three typical spectra obtained with the detector under the respective conditions: no neutron source, converters installed (area 1); with neutron source but no converter (area 2); with neutron source, converters installed (area 3). The gamma contribution to the spectrum is roughly below $0.6 \mathrm{MeV}$; the alpha contribution between $0.6 \mathrm{MeV}$ and $1.7 \mathrm{MeV}$; the triton contribution between $1.7 \mathrm{MeV}$ and $2.7 \mathrm{MeV}$.

of respectively $\approx 1 \%$ and $\approx 0.85 \%$. In case of two identical $1.8 \mu$ m converters the intrinsic efficiency would be $2 \%$. As for the detection efficiency, it depends on the energy loss of the two reaction products inside the converter itself and in the dead layer of the silicon detector, and on the electronic threshold. An accurate calculation was performed in [8], giving a value around $75 \%$. Under the described configuration a thermal neutron impinging on the detector will have $\mathrm{a} \approx 1.5 \%$ probability of being detected. In order to increase this probability one will have to increase the overall active area, either tiling or stacking several identical detector sandwich assemblies.

\section{Scintillators for neutron detection}

A technique similar to the one described in the previous section can be fruitfully applied to scintillators. Due to the usually lower cost of scintillators one can decide to deposit the converter layer directly on the scintillator itself or to make use of an additional substrate. For our first prototypes we have been using tiny strips of $\mathrm{CsI}(\mathrm{Tl})$ as scintillator, either with direct deposition of $\mathrm{LiF}$ or by wrapping them with an aluminum foil onto which the converter was deposited [9].

We will show here a few preliminary results obtained with a special strip configuration which we set up, in order to prove the position sensitivity of such a detector. The strip size was $25 \mathrm{~mm} \times 3 \mathrm{~mm} \times$ $1 \mathrm{~mm}$, and it was first wrapped with a few tiny aluminum+converter strips. These strips were spaced $2 \mathrm{~mm}$ from one another, and afterward the detector was wrapped in a simple aluminum foil acting as a reflector (Fig. 5).

The scintillation light readout was performed by means of two silicon photomultipliers (SiPM) optically coupled to the two strip ends. The detector was exposed to a moderated AmBe neutron source, and the data were analyzed using the same technique as described in $[10,11]$ that also allows to reconstruct the impact position on the strip. The total light spectrum, shown in Fig. 6, has a clear peak indicating the detection of tritons, which is the signature of a neutron capture in the converter. Unfortunately the alpha peak is hidden inside the exponential gamma contribution, due to the lower light yield for heavier particles, intrinsic of the scintillators [12]. By building a 3D spectrum having the 


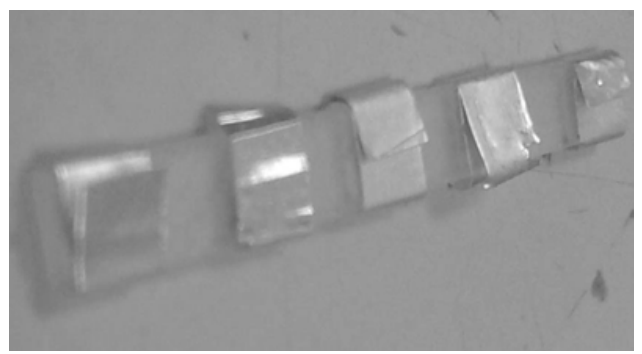

Figure 5. A $25 \mathrm{~mm} \times 3 \mathrm{~mm} \times 1 \mathrm{~mm} \mathrm{CsI(Tl)} \mathrm{scintillator} \mathrm{strip} \mathrm{wrapped} \mathrm{with} \mathrm{five} \mathrm{neutron} \mathrm{converter} \mathrm{layers} \mathrm{deposited}$ onto aluminum foils. This detector was then wrapped in a simple aluminum foil acting as a reflector.

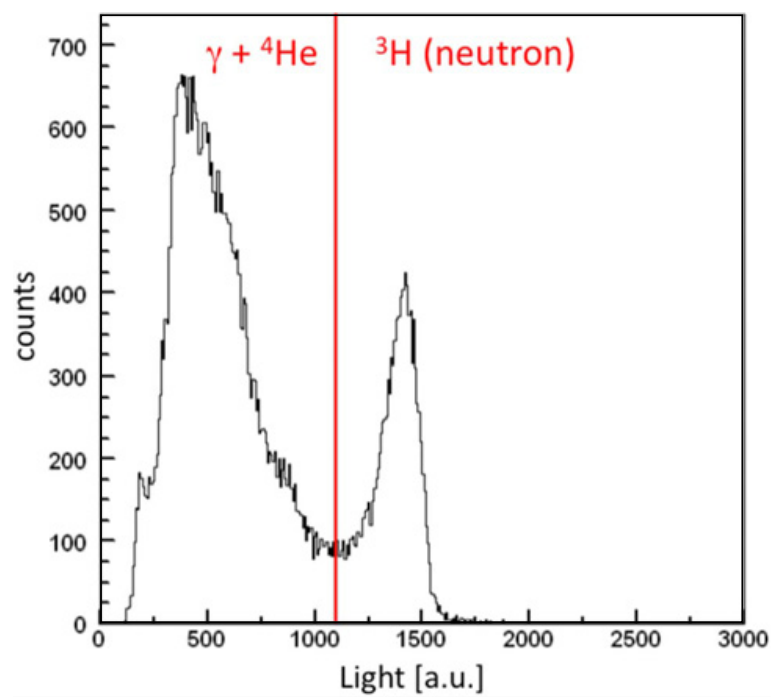

Figure 6. The total light spectrum obtained by means of the detector of Fig. 5 when exposed to the moderated $\mathrm{AmBe}$ neutron source. A clear peak indicating the detection of tritons is the signature of neutrons.

left and right light output from the strip on the $\mathrm{X}$ and $\mathrm{Y}$ axis, under the condition that the total light were under the triton peak, one can immediately see the effect of the neutron converter strips placed on the scintillator (Fig. 7). The spatial resolution we evaluated from the current data is around $1 \mathrm{~mm}$.

We have worked out several improvements of this technique (for which we have a patent pending) with different detector and converter geometries and size, and using different scintillators; at the moment we have several ongoing tests with quite encouraging preliminary results [9].

\section{Envisaged applications}

We expect that the use of ${ }^{6} \mathrm{LiF}$, in suitable arrangement onto several substrates, could represent a viable replacement of ${ }^{3} \mathrm{He}$ in many applications. Tiny scintillator strips could even represent a considerable improvement for applications at neutron beam facilities, due to their good spatial resolution. Using larger scintillators and/or tiling silicon pad detectors, suitably coupled with neutron converters, could be used for wider solid angle coverage and efficiency, as required for instance in homeland security.

This is why we are already working at two possible applications. The first one is a set of panels, neutron sensitive, to be placed around neutron emitting material in a $4 \pi$ solid angle coverage for 


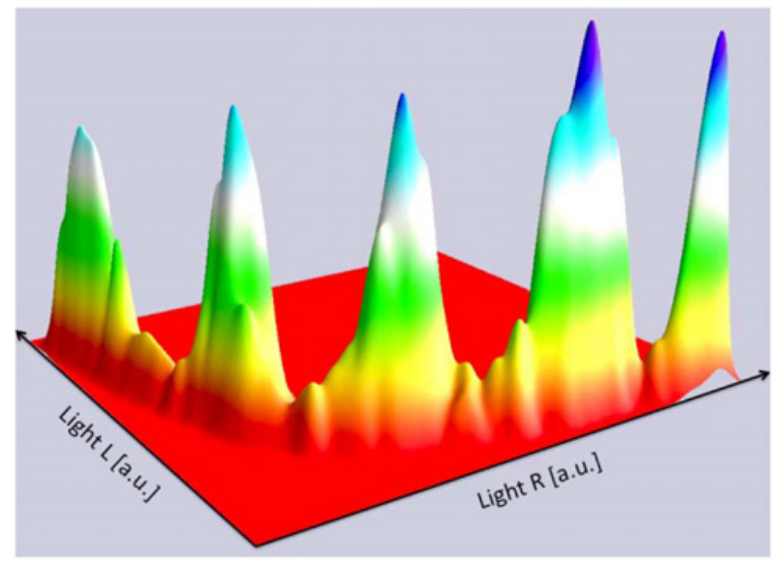

Figure 7. 3D spectrum with left and right light output from the strip on the $\mathrm{X}$ and $\mathrm{Y}$ axis, under the condition that the total light were under the triton peak. The effect of the neutron converter strips placed on the scintillator is clearly seen.

coincidence neutron counting, exploiting a well known technique to measure the amount of fissile material in a sample [14].

The second application we are pursuing is the extension of the DMNR system [13] we already developed for the online monitoring of radioactive waste repositories. In the new configuration such a system would also be equipped with a large number of small neutron detectors, capable of ensuring the continuity of knowledge on spent fuel rods sealed inside their casks. This is currently a relevant issue, as it is aimed at preventing the diversion of fuel elements for illegal or, worse, weapon use.

\section{Conclusion}

We have developed a low-cost technique to build thermal neutron detectors, based on ${ }^{6} \mathrm{LiF}$ converters coupled to particle detectors, likely useful in several applications as a replacement of the traditional ${ }^{3} \mathrm{He}$ tubes. We are actively working on further improvements, in particular making use of scintillators, in order to increase the intrinsic (several layers) and the geometrical (wider area) efficiency. The preliminary results we obtained look very promising, especially in view of a possible application for the online real time monitoring of spent fuel casks, in order to ensure the continuity of knowledge for safety and security purposes.

In this respect we report a sentence from ref [15]: "The future evolution of requirements and technologies might bring important impacts on cask operations and managements for spent fuel storage facilities", and this is what we think is just going to happen.

We are indebted to Paolo Peerani and Vittorio Forcina of the European Commission, Joint Research Centre, Institute of Transuranium Elements, Ispra, Italy, for making available the AmBe neutron source and the moderator. Apart from that, their advice and suggestions were invaluable.

\section{References}

[1] C. Manfredotti, A. Lo Giudice, F. Fasolo, E. Vittone, C. Paolini, F. Fizzotti, A. Zanini, G. Wagner, C. Lanzieri, Nucl. Instr. And Meth. A552, 131-137 (2005)

[2] R. J. Nikolic, C. L. Cheung, C. E. Reinhardt, T. F. Wang, Nanotech 2006 Boston United State, UCRL-PROC-219274 (2006) 
[3] D.S. McGregor, M. D. Hammig, Y. H. Yang, H. K. Gersch, R.T. Klann, Nucl. Instr. And Meth. A500, 272-308 (2003)

[4] C. A. Baker, K. Green, M. G. D. van der Grinten, P. S. Iaydjiev, S. N. Ivanov, S. Al-Ayoubi, P. G. Harris, J. M. Pendlebury, D. B. Shiers, P. Geltenbort, Nucl. Instr. And Meth.in Phys. Res. A487, 511-520 (2002)

[5] B. F. Phlips, F. J. Kub, E. I. Novikova, E. A. Wulf, C. Fitzgerald, Nucl. Instr. And Meth. A579, 173-176 (2007)

[6] J. Uher, C. Fröjdh, J. Jakubek, C. Kenney, Z. Kohout, V. Linhart, S. Parker, S. Petersson, S. Pospìsil, G. Thungström, Nucl. Instr. And Meth. A576, 32-37 (2007)

[7] M. Voytchev, M. P. Iñiguez, R. Méndez, A. Mañanes, L. R. Rodrìguez, R. Barquero, Nucl. Instr. And Meth. A512, 546-552 (2003)

[8] M. Barbagallo, L. Cosentino, V. Forcina, C. Marchetta, A. Pappalardo, P. Peerani, C. Scirè, S. Scirè, M. Schillaci, S. Vaccaro, G. Vecchio, P. Finocchiaro, Rev. Sci. Instrum. 84, 033503 (2013)

[9] P. Finocchiaro, M. Barbagallo, L. Cosentino, C. Marchetta, A. Pappalardo, patent pending RM2013A000254

[10] L. Cosentino, P. Finocchiaro, A. Pappalardo, F. Garibaldi, Rev. Sci. Instrum. 83, 084302 (2012)

[11] L. Cosentino, P. Finocchiaro, A. Pappalardo, F. Garibaldi, Rev. Sci. Instrum. 83, 114301 (2012)

[12] J. Alarja, A. Dauchy, A. Giorni, C. Morand, E. Pollaco, P. Stassi, R. Billerey, B. Chambon, B. Cheynis, D. Drain, C. Pastor, Nucl. Instr. And Meth. A242, 352-354 (1986)

[13] P. Finocchiaro, in Radioactive Waste: Sources, Types and Management, Nova Science Publishers, (2011)

[14] N. Ensslin, in Passive Nondestructive Assay of Nuclear Materials, NUREG/CR-5550 LA-UR-90732, 457 (1991)

[15] Operation and Maintenance of Spent Fuel Storage and Transportation Casks/Containers, IAEATECDOC-1532 (2007) 